\title{
Racial Discrimination in Health Care and Utilization of Health Care: a Cross-sectional Study of California Adults
}

\author{
Héctor E. Alcalá, MPH, PhD and Daniel M. Cook, $P h D^{2}$ \\ 'Department of Family, Population and Preventive Medicine, Program in Public Health, Stony Brook University, Stony Brook, NY, USA; ${ }^{2}$ School of \\ Community Health Sciences, University of Nevada, Reno, Reno, NV, USA.
}

\begin{abstract}
BACKGROUND: Racial and ethnic discrimination in health care have been associated with suboptimal use of health care. However, limited research has examined how facets of health care utilization influence, and are influenced by, discrimination.
\end{abstract}

OBJECTIVE: This study aimed to determine if type of insurance coverage and location of usual source of care used were associated with perceptions of racial or ethnic discrimination in health care. Additionally, this study examined if perceived racial or ethnic discrimination influenced delaying or forgoing prescriptions or medical care. DESIGN: Data from the 2015-2016 California Health Interview Survey were used. Logistic regression models estimated odds of perceiving racial or ethnic discrimination from insurance type and location of usual source of care. Logistic regression models estimated odds of delaying or forgoing medical care or prescriptions.

PARTICIPANTS: Responses for 39,171 adults aged 18 and over were used.

MAIN MEASURES: Key health care utilization variables were as follows: current insurance coverage, location of usual source of care, delaying or forgoing medical care, and delaying or forgoing prescriptions. We examined if these effects differed by race. Ever experiencing racial or ethnic discrimination in the health care setting functioned as a dependent and independent variable in analyses.

KEY RESULTS: When insurance type and location of care were included in the same model, only the former was associated with perceived discrimination. Specifically, those with Medicaid had 66\% higher odds of perceiving discrimination, relative to those with employer-sponsored coverage (AOR $=1.66 ; 95 \%$ CI $1.11,2.47$ ). Race did not moderate the impact of discrimination. Perceived discrimination was associated with higher odds of delaying or forgoing both prescriptions (AOR $=1.97$; 95\% CI 1.26, 3.09) and medical care (AOR $=1.84 ; 95 \%$ CI 1.31, 2.59).

CONCLUSIONS: Health care providers have an opportunity to improve the experiences of their patients, particularly those with publicly sponsored coverage.

KEY WORDS: health insurance; race; discrimination; health care utilization.

J Gen Intern Med 33(10): 1760-7

DOI: $10.1007 / \mathrm{s} 11606-018-4614-4$

(C) Society of General Internal Medicine 2018

Received January 11, 2018

Revised June 5, 2018

Accepted July 19, 2018

Published online August 8, 2018

\section{INTRODUCTION}

Experiencing or perceiving racial or ethnic discrimination is associated with negative health consequences, including mortality, mental illness, cancer, hypertension, cardiovascular disease, obesity, and risky health behaviors. ${ }^{1-6}$ Reasons for these associations are multifaceted and involve cognitive, emotional, and biological processes. ${ }^{7}$ Consequently, it is unsurprising that discrimination among minority groups is one of the reasons for the enduring patterns of racial and ethnic health disparities in the United States (US) ${ }^{8}$

Racial discrimination can impact health by leading to suboptimal use of health care. ${ }^{9}$ Experiences of discrimination in the health care setting negatively impact the use of a variety of health services, including cancer screening, ${ }^{10}$ pharmacy services, ${ }^{11}$ and needed medical and mental health care. ${ }^{12}$ Those who report racial or ethnic discrimination in health care are more likely to delay filling a prescription, but not more likely to delay obtaining medical care, relative to those not reporting discrimination. ${ }^{11}$ Similarly, experiences of discrimination outside of the health care setting are associated with suboptimal use of health care. ${ }^{13}$ Collectively, the negative impact of discrimination is not surprising when considering that individuals reporting discrimination in health care also report higher rates of problems with the care they receive than those who do not, ${ }^{14}$ creating disincentives for continued use of care.

Previous experiences of racial discrimination erode trust in the health care system ${ }^{11}$ and thus shape or discourage future interactions with the health care system. Overall, racial minorities have lower trust in the health care system, relative to non-Latino whites. ${ }^{15}$ Extant research has shown that many different racial minority groups report higher rates of discrimination in the health care setting, than do non-Latino whites, even after accounting for a variety of confounders. ${ }^{16-19}$ These patterns of discrimination are important because they account for racial differences in perceived quality of health care between blacks and whites ${ }^{20}$ and differences in provider trust ${ }^{21}$ suggesting that experiences of discrimination play a major role in the subjective evaluation of one's health care. Furthermore, those with previous experiences of racial discrimination have greater apprehension with providing race/ethnicity information to hospital staff, ${ }^{22}$ thus potentially undermining our 
understanding of racial and ethnic health disparities by hindering the collection of needed data. Given the importance of discrimination and utilization of health care, the present study had three overall objectives: (1) Examine which characteristics of health care utilization (particularly insurance type and location of usual source of care) are associated with perceiving discrimination in health care; (2) determine if these associations vary by race; and (3) examine if perceiving discrimination is associated with delays in receiving needed medical care.

Exposure to discrimination in health care varies by factors outside of race and ethnicity. For example, those who get their health care in a setting that is not a doctor's office (i.e., clinic, emergency room, etc.) are more likely to report discrimination in health care, relative to those receiving care in a doctor's office. ${ }^{23}$ However, this research has not specifically examined how emergency room usage impacts reports of discrimination, since it categorized it with other sources of care. Additionally, there is a paucity of research examining how insurance type can impact exposure to racial and ethnic discrimination in health care. This is crucial as the Patient Protection and Affordable Care Act (ACA) has increased the percent of Americans with health insurance ${ }^{24}$ through various mechanisms. In particular, Medicaid expansion extended eligibility to more Americans by expanding income eligibility thresholds and eliminating other barriers, and the ACA insurance exchanges allowed Americans to buy private insurance plans with the help of government subsidies. ${ }^{25-27}$ However, changes to insurance offerings and coverage introduced by the ACA did not eliminate disparities in access to care by insurance type. ${ }^{28}$ For example, one recent study noted the varying size of insurance plans under the law, and found that Latinos were disproportionately in narrower plans with less choice of providers. ${ }^{29}$ Thus, the changing menu of insurance options and influx of newly insured patients into the health care setting make it important to determine how insurance type is associated with experiences of racial or ethnic discrimination in health care.

This study examined the California context, which is a racially diverse state and had a more generous expansion of Medicaid eligibility than other states. ${ }^{30}$ Furthermore, there is mixed evidence on the ACA's ability to attenuate racial and ethnic disparities in health care utilization, suggesting considerable barriers to equitable care remain. ${ }^{31,32}$ As such, differences in perceived racial or ethnic discrimination of health care by insurance type or location of health care can reflect both differences in the populations served (in terms of their prior history with discrimination) and the quality of care they are receiving in the ACA era, both of which are important questions to consider when evaluating the quality of and access to health care. Finally, examining how perceived discrimination impacts the use of health care services can help us understand the extent to which these remained a barrier prior to any repeal of ACA provisions.

\section{METHODS}

\section{Data Source}

Data come from the 2015 and 2016 Adult California Health Interview Survey (CHIS). This annual telephone survey is administered to adults (18 and over), residing in households. ${ }^{33}$ Respondents were obtained using random-digit dial of both cellphones and landlines as well as Korean and Japanese surname lists. ${ }^{33}$ Surveys were administered in English, Cantonese, Korean, Mandarin, Spanish, Tagalog, and Vietnamese. Missing data for most variables was imputed by CHIS researchers. ${ }^{33} \mathrm{~A}$ total of 42,089 respondents completed the CHIS. After excluding cases with missing data (i.e., missing in error, do not know, or refused) on any study variable, 41,950 respondents remained. The study was further restricted to the 39,171 who were currently insured. Of these, 132 interviews were completed by proxies on behalf of the respondent. The remaining interviews were completed by respondents.

\section{Variables}

Two independent variables captured characteristics of a respondent's health care utilization. The first consisted of the respondent's current type of health insurance coverage. Types of insurance consisted of the following: (1) employer-sponsored coverage; (2) private insurance coverage purchased through Covered California (i.e., the health insurance exchange in California); (3) private insurance coverage not purchased through Covered California (i.e., the open-market/off-exchange); (4) Medicaid; (5) Medicare and Medicaid in combination; (6) Medicare alone or in combination with any other nonMedicaid insurance; and (7) other publically funded insurance programs. While employer-sponsored coverage is a type of private insurance, we separate it from privately purchased coverage obtained through the individual market, because different categories of private insurance coverage have been shown to differentially impact access to health care. ${ }^{28}$ Employersponsored coverage served as the reference category in analyses because this insurance type is associated with fewer barriers to health care and is the most common insurance type among Californians. ${ }^{28}$ The second variable captured where the respondent went for their usual source of health care. This was coded into five categories: (1) doctor's office, HMO, or Kaiser; (2) no usual source of care; (3) clinic, health center, or hospital clinic; (4) emergency room; or (5) other place or no one place.

Two dependent variables measured inability to get needed health care. The first was a dichotomous measure indicating if the respondent had delayed or forgone needed prescription drugs in the past 12 months for a reason other than costs or lack of insurance. The second was a dichotomous measure indicating if the respondent had delayed or forgone needed medical care in the past 12 months for a reason other than costs or lack of insurance.

Perceived racial or ethnic discrimination in health care functioned as an independent and dependent variable in analyses. Respondents were asked, "Was there ever a time when 
you would have gotten better medical care if you had belonged to a different race or ethnic group?" Respondents who answered in the affirmative were coded as having perceived racial or ethnic discrimination. This coding scheme is consistent with previous research using the CHIS. ${ }^{17,} 20$

Several variables were included in multivariate analyses as potential confounders. Gender, educational attainment (bachelor's degree or above versus less than bachelor's degree), limited English proficiency (yes versus no) health status (excellent, very good, or good versus fair or poor), lifetime diagnoses of a chronic health condition (yes or no), and urban or rural residence were measured as dichotomous indicators. Lifetime diagnoses of chronic health condition was based on whether or not the respondent reported ever being diagnosed with asthma, diabetes, high blood pressure, or heart disease. Race/ethnicity (white, Latino, black, Asian, and other), citizenship status (US-born citizen, naturalized citizen, or noncitizen), age (18-29, 30-39, 40-49, 50-59, 60-69, or 70 and over), and income as a percent of the federal poverty level (FPL; 0-138\%, 139-249\%, 250-399\%, and 400\% or higher) were measured using categorical variables.

\section{Analyses}

All analyses were conducted in Stata 14.2 and made use of jackknife replicate weights to yield robust standard errors and ensure estimates were representative of the underlying population. Univariate statistics were calculated for each of the study subsamples. Then, chi-squared tests were used to determine if the distribution of sample characteristic differed by perceived discrimination. Logistic regressions were used to calculate odds of reporting perceived discrimination, while accounting for potential confounders. In these models, insurance type and location of usual source of health care functioned as the independent variables. The variables were included in separate models, and then included in one model simultaneously. Post hoc tests examined differences between plans purchased through Covered California and those purchased off-exchange. In order to determine if any of these factors were dependent on respondent race, an interaction term between each independent variable and race was tested. Because no interaction terms were significant, only the summary of the test for interaction is presented.

Finally, logistic regression models were used to calculate odds of delaying or forgoing prescriptions or medical care in the past 12 months. In these models, perceived discrimination was the independent variable and all control variables, insurance type, and location of usual source of health care were accounted for.

\section{RESULTS}

Table 1 shows the sample characteristics. Over $6 \%$ of respondents perceived racial or ethnic discrimination in healthcare. The plurality of respondents had health insurance coverage through their employers. The plurality of respondents were non-Latino white, between the ages of 18 and 29, lived in households at or above $400 \%$ of the FPL, and had not been diagnosed with a chronic health condition or were in good health. The majority of respondents were US-born, proficient in English, female, had less than a bachelor's degree, or resided in an urban area.

Table 1 also shows the distribution of sample characteristics, by perceived discrimination. Insurance type, delaying or forgoing prescriptions, location of usual source of care, race/ethnicity, citizenship status, English proficiency, age, household income, urban or rural residence, and health status were each associated with perceiving racial or ethnic discrimination in health care.

Table 2 shows the odds of perceiving discrimination in health care. In model 1 , those with Medicaid $(\mathrm{AOR}=1.71$; $95 \%$ CI 1.19, 2.60), Medicare and Medicaid $(\mathrm{AOR}=1.83$; $95 \%$ CI 1.06, 3.17), and Medicare alone or with other insurance $(\mathrm{AOR}=1.72 ; 95 \%$ CI $1.02,2.92)$ had higher odds of perceiving discrimination, relative to those with employersponsored coverage. In post hoc tests (not shown), those with private coverage purchased through Covered California did not differ in their odds of perceiving discrimination, when compared to those with private coverage purchased offexchange (AOR $=2.01 ; 95 \%$ CI $0.76,5.33)$. The interaction with race was not significant $(p=0.0635)$. In model 2 , reporting the emergency room as one's usual source of health care was associated with twice the odds of perceiving discrimination, relative to those with employer-sponsored coverage $(\mathrm{AOR}=1.91 ; 95 \% \mathrm{CI} 1.07,3.42)$. The interaction with race was not significant $(p=0.6541)$. In model 3 , those with Medicaid (AOR $=1.66 ; 95 \%$ CI 1.11, 2.47), Medicare and Medicaid $(\mathrm{AOR}=1.80 ; 95 \% \mathrm{CI} 1.03,3.14)$, and Medicare alone or with other insurance $(\mathrm{AOR}=1.71 ; 95 \%$ CI $1.01,2.89)$ had higher odds of perceiving discrimination, relative to those with employer-sponsored coverage. Location of usual source of care was not associated with discrimination in this model.

Table 3 shows the odds of delaying or forgoing prescriptions or medical care in the past 12 months, for reasons other than being uninsured or costs. Perceiving racial or ethnic discrimination in health care was associated with nearly twice the odds of delaying or forgoing prescriptions $(\mathrm{AOR}=1.97$; $95 \%$ CI $1.26,3.09$ ) and $84 \%$ higher odds of delaying or forgoing medical care $(\mathrm{AOR}=1.84 ; 95 \%$ CI $1.31,2.59)$ in the past 12 months.

\section{DISCUSSION}

The present study highlighted important disparities in perceiving discrimination in health care. Consistent with previous work, non-whites are far more likely to report discrimination in health care. ${ }^{16-19}$ Also, consistent with a prior study in California, ${ }^{23}$ the location of usual source of health care is associated with perceived racial or ethnic discrimination in 
Table 1 Sample Characteristics, CHIS 2015-2016 $(N=39,171)$

\begin{tabular}{|c|c|c|c|c|c|c|c|c|}
\hline & \multicolumn{3}{|c|}{ All respondents } & \multicolumn{5}{|c|}{ Experienced discrimination in health care } \\
\hline & \multirow[b]{2}{*}{$N$} & \multirow[b]{2}{*}{$\%$} & \multirow[b]{2}{*}{ SE } & \multicolumn{2}{|l|}{ No } & \multicolumn{2}{|l|}{ Yes } & \multirow[t]{2}{*}{$P$ value chi-squared } \\
\hline & & & & $\%$ & SE & $\%$ & SE & \\
\hline \multicolumn{9}{|c|}{ Perceived racial or ethnic discrimination in health care } \\
\hline No & 37,022 & 93.7 & 0.28 & - & - & - & - & \\
\hline Yes & 2149 & 6.3 & 0.28 & - & - & - & - & \\
\hline \multicolumn{8}{|l|}{ Type of insurance coverage } & $<.0001$ \\
\hline Employer-sponsored coverage & 13,420 & 46.14 & 0.54 & 46.51 & 0.56 & 40.64 & 2.47 & \\
\hline Medicaid & 7108 & 24.45 & 0.53 & 23.41 & 0.53 & 39.94 & 2.74 & \\
\hline Private coverage, Covered California & 1167 & 3.43 & 0.27 & 3.47 & 0.29 & 2.83 & 0.80 & \\
\hline Private coverage, off-exchange & 1340 & 4.07 & 0.27 & 4.19 & 0.28 & 2.27 & 0.78 & \\
\hline Medicare and Medicaid & 3649 & 6.04 & 0.22 & 6 & 0.22 & 6.56 & 1.36 & \\
\hline Medicare alone or with other insurance & 12,004 & 14.41 & 0.21 & 14.93 & 0.24 & 6.71 & 0.99 & \\
\hline Other public-sponsored coverage & 483 & 1.46 & 0.16 & 1.49 & 0.17 & 1.06 & 0.45 & \\
\hline Delayed or did not get needed prescription & jast $12 \mathrm{~m}$ & & & & & & & 0.019 \\
\hline No & 36,793 & 93.97 & 0.27 & 94.18 & 0.28 & 90.97 & 1.58 & \\
\hline Yes & 2378 & 6.03 & 0.27 & 5.82 & 0.28 & 9.03 & 1.58 & \\
\hline Delayed or did not get needed medical car & past $12 \mathrm{n}$ & ths & & & & & & 0.1204 \\
\hline No & 36,296 & 92.72 & 0.37 & 92.85 & 0.39 & 90.88 & 0.13 & \\
\hline Yes & 2875 & 7.28 & 0.37 & 7.15 & 0.39 & 9.12 & 0.13 & \\
\hline Location of usual source of care & & & & & & & & 0.0101 \\
\hline Doctor's office, HMO, or Kaiser & 24,758 & 57.88 & 0.55 & 58.48 & 0.56 & 48.9 & 2.72 & \\
\hline No usual source of care & 3553 & 12.5 & 0.39 & 12.34 & 0.39 & 14.91 & 2.06 & \\
\hline Clinic or health center & 9927 & 26.88 & 0.5 & 26.53 & 0.5 & 32.21 & 2.48 & \\
\hline Emergency room & 508 & 1.62 & 0.19 & 1.56 & 0.19 & 2.42 & 0.58 & \\
\hline Other or no one place & 425 & 1.12 & 0.15 & 1.09 & 0.15 & 1.56 & 0.72 & \\
\hline Race/ethnicity & & & & & & & & $<.0001$ \\
\hline White & 22,720 & 43.94 & 0.24 & 46.01 & 0.33 & 13.23 & 0.24 & \\
\hline Latino & 8755 & 32.39 & 0.29 & 31.6 & 0.36 & 44.13 & 0.29 & \\
\hline Black & 2119 & 5.8 & 0.07 & 4.87 & 0.13 & 19.55 & 1.69 & \\
\hline Asian & 4112 & 14.72 & 0.15 & 14.56 & 0.21 & 17.09 & 2.04 & \\
\hline Other & 1465 & 3.15 & 0.08 & 2.96 & 0.1 & 6 & 0.95 & \\
\hline Citizenship status & & & & & & & & 0.0001 \\
\hline US-born citizen & 30,053 & 68.38 & 0.44 & 69.01 & 0.5 & 59.04 & 2.26 & \\
\hline Naturalized citizen & 5890 & 18.31 & 0.48 & 17.94 & 0.5 & 23.87 & 2.01 & \\
\hline Non-citizen & 3228 & 13.31 & 0.45 & 13.06 & 0.46 & 17.1 & 1.71 & \\
\hline Limited English proficiency & & & & & & & & 0.0076 \\
\hline No & 32,221 & 76.04 & 0.51 & 76.44 & 0.52 & 70.12 & 2.46 & \\
\hline Yes & 6950 & 23.96 & 0.51 & 23.56 & 0.52 & 29.88 & 2.46 & \\
\hline Gender & & & & & & & & 0.4783 \\
\hline Male & 16,700 & 47.63 & 0.24 & 47.74 & 0.28 & 45.85 & 0.24 & \\
\hline Female & 22,471 & 52.38 & 0.24 & 52.26 & 0.28 & 54.15 & 0.24 & \\
\hline Educational attainment & & & & & & & & 0.1802 \\
\hline Less than bachelor's degree & 22,930 & 60.66 & 0.47 & 60.43 & 0.47 & 64.06 & 0.26 & \\
\hline Bachelor's degree or above & 16,241 & 39.34 & 0.47 & 39.57 & 0.47 & 35.94 & 0.26 & \\
\hline Age & & & & & & & & $<.0001$ \\
\hline $18-29$ & 4804 & 21.1 & 0.2 & 20.94 & 0.26 & 23.44 & 2.04 & \\
\hline $30-39$ & 3720 & 17.59 & 0.21 & 17.37 & 0.24 & 20.82 & 2.06 & \\
\hline $40-49$ & 4434 & 17.04 & 0.2 & 16.91 & 0.23 & 19.04 & 1.82 & \\
\hline $50-59$ & 6958 & 16.37 & 0.31 & 16.14 & 0.32 & 19.83 & 1.69 & \\
\hline $60-69$ & 8832 & 15.31 & 0.34 & 15.59 & 0.38 & 11.15 & 1.16 & \\
\hline $70+$ & 10,423 & 12.59 & 0.19 & 13.05 & 0.21 & 5.72 & 1.09 & \\
\hline Household income (as \% of federal povert & $\operatorname{vel}(\mathrm{FPL}))$ & & & & & & & $<.0001$ \\
\hline $0-138 \%$ of FPL & 9445 & 25.53 & 0.55 & 24.82 & 0.56 & 36.17 & 2.19 & \\
\hline $139-249 \%$ of FPL & 6608 & 16.77 & 0.47 & 16.6 & 0.48 & 19.31 & 1.66 & \\
\hline $250-399 \%$ of FPL & 6345 & 16.56 & 0.49 & 16.45 & 0.51 & 18.22 & 1.99 & \\
\hline $400 \%$ or greater of FPL & 16,773 & 41.13 & 0.57 & 42.13 & 0.61 & 26.29 & 2.29 & \\
\hline Urban or rural residence & & & & & & & & 0.0074 \\
\hline Rural & 7232 & 9.89 & 0.26 & 10.11 & 0.26 & 6.54 & 1.06 & \\
\hline Urban & 31,939 & 90.11 & 0.26 & 89.89 & 0.26 & 93.46 & 1.06 & \\
\hline Health status & & & & & & & & 0.0001 \\
\hline Excellent, very good, or good & 30,300 & 79.33 & 0.47 & 79.7 & 0.5 & 73.72 & 0.15 & \\
\hline Fair or Poor & 8871 & 20.67 & 0.47 & 20.3 & 0.5 & 26.28 & 0.15 & \\
\hline Chronic condition & & & & & & & & 0.5691 \\
\hline No & 18,251 & 56.01 & 0.61 & 56.1 & 0.65 & 54.6 & 2.49 & \\
\hline Yes & 20,920 & 43.99 & 0.61 & 43.9 & 0.65 & 45.4 & 2.49 & \\
\hline Survey year & & & & & & & & 0.6021 \\
\hline 2015 & 19,561 & 49.64 & 0.22 & 49.56 & 0.29 & 50.82 & 2.22 & \\
\hline 2016 & 19,610 & 50.36 & 0.22 & 50 & 0.29 & 49.18 & 2.22 & \\
\hline
\end{tabular}

All frequencies and standard errors are weighted. Sample sizes are unweighted

health care. However, unlike prior research, we were able to show those receiving care in the emergency room had the highest reports of perceived discrimination. ${ }^{23}$ As the prior study relied on data that was released prior to ACA 
Table 2 Logistic Regression Models for Odds of Perceiving Racial or Ethnic Discrimination $(N=39,171)$

\begin{tabular}{|c|c|c|c|c|c|c|}
\hline & \multicolumn{2}{|l|}{ Model 1} & \multicolumn{2}{|l|}{ Model 2} & \multicolumn{2}{|l|}{ Model 3} \\
\hline & AOR & $95 \% \mathrm{CI}$ & AOR & $95 \% \mathrm{CI}$ & AOR & $95 \% \mathrm{CI}$ \\
\hline \multicolumn{7}{|l|}{ Type of insurance coverage } \\
\hline Employer-sponsored coverage & Reference & & Reference & & Reference & \\
\hline Medicaid & 1.71 & $(1.19,2.60)$ & - & - & 1.66 & $(1.11,2.47)$ \\
\hline Private coverage, Covered California & 1.89 & $(0.90,4.01)$ & - & - & 1.86 & $(0.87,3.96)$ \\
\hline Private coverage, off-exchange & 0.94 & $(0.49,1.80)$ & - & - & 0.93 & $(0.48,1.78)$ \\
\hline Medicare and Medicaid & 1.83 & $(1.06,3.17)$ & - & - & 1.80 & $(1.03,3.14)$ \\
\hline Medicare alone or with other insurance & 1.72 & $(1.02,2.92)$ & - & - & 1.71 & $(1.01,2.89)$ \\
\hline Other public-sponsored coverage & 1.91 & $(0.52,7.02)$ & - & - & 1.85 & $(0.48,7.03)$ \\
\hline \multicolumn{7}{|l|}{ Location of usual source of care } \\
\hline Doctor's office, HMO, or Kaiser & Reference & & Reference & & Reference & \\
\hline No usual source of health care & - & - & 1.25 & $(0.84,1.88)$ & 1.18 & $(0.79,1.77)$ \\
\hline Clinic or health center & - & - & 1.13 & $(0.81,1.56)$ & 1.05 & $(0.76,1.46)$ \\
\hline Emergency room & - & - & 1.91 & $(1.07,3.42)$ & 1.72 & $(0.94,3.12)$ \\
\hline Other or no one place & - & - & 1.66 & $(0.68,4.06)$ & 1.58 & $(0.64,3.90)$ \\
\hline \multicolumn{7}{|l|}{ Race/ethnicity } \\
\hline White & Reference & & Reference & & Reference & \\
\hline Latino & 1.80 & $(1.17,2.76)$ & 1.75 & $(1.14,2.70)$ & 1.79 & $(1.16,2.75)$ \\
\hline Black & 4.72 & $(3.00,7.41)$ & 4.75 & $(3.01,7.48)$ & 4.71 & $(2.98,7.42)$ \\
\hline Asian & 1.66 & $(1.03,2.68)$ & 1.63 & $(1.01,2.63)$ & 1.66 & $(1.03,2.68)$ \\
\hline Other & 2.63 & $(1.56,4.44)$ & 2.65 & $(1.57,4.47)$ & 2.64 & $(1.56,4.46)$ \\
\hline \multicolumn{7}{|l|}{ Citizenship status } \\
\hline US-born citizen & Reference & & Reference & & Reference & \\
\hline Naturalized citizen & 1.13 & $(0.69,1.85)$ & 1.11 & $(0.67,1.82)$ & 1.13 & $(0.69,1.86)$ \\
\hline Non-citizen & 1.03 & $(0.64,1.64)$ & 1.01 & $(0.63,1.63)$ & 1.02 & $(0.63,1.64)$ \\
\hline \multicolumn{7}{|l|}{ Limited English proficiency } \\
\hline No & Reference & & Reference & & Reference & \\
\hline Yes & 0.98 & $(0.65,1.47)$ & 0.97 & $(0.65,1.46)$ & 0.98 & $(0.65,1.46)$ \\
\hline \multicolumn{7}{|l|}{ Gender } \\
\hline Male & Reference & & Reference & & Reference & \\
\hline Female & 1.17 & $(0.91,1.51)$ & 1.23 & $(0.96,1.57)$ & 1.20 & $(0.94,1.53)$ \\
\hline \multicolumn{7}{|l|}{ Educational attainment } \\
\hline Less than bachelor's degree & Reference & & Reference & & Reference & \\
\hline Bachelor's degree or above & 1.24 & $(0.85,1.82)$ & 1.26 & $(0.83,1.80)$ & 1.25 & $(0.85,1.83)$ \\
\hline \multicolumn{7}{|l|}{ Age } \\
\hline $18-29$ & Reference & & Reference & & Reference & \\
\hline $30-39$ & 1.15 & $(0.74,1.78)$ & 1.17 & $(0.76,1.80)$ & 1.17 & $(0.76,1.80)$ \\
\hline $40-49$ & 1.04 & $(0.69,1.57)$ & 1.04 & $(0.68,1.61)$ & 1.07 & $(0.70,1.61)$ \\
\hline $50-59$ & 1.00 & $(0.65,1.56)$ & 1.03 & $(0.67,1.60)$ & 1.04 & $(0.67,1.61)$ \\
\hline $60-69$ & 0.68 & $(0.46,0.99)$ & 0.78 & $(0.55,1.14)$ & 0.70 & $(0.48,1.03)$ \\
\hline $70+$ & 0.31 & $(0.13,0.77)$ & 0.41 & $(0.17,0.97)$ & 0.32 & $(0.13,0.81)$ \\
\hline \multicolumn{7}{|c|}{ Household income (as \% of federal poverty level (FPL)) } \\
\hline $0-138 \%$ of FPL & Reference & & Reference & & Reference & \\
\hline $139-249 \%$ of FPL & 0.88 & $(0.60,1.30)$ & 0.80 & $(0.56,1.15)$ & 0.89 & $(0.61,1.31)$ \\
\hline $250-399 \%$ of FPL & 0.80 & $(0.48,1.34)$ & 0.68 & $(0.43,1.05)$ & 0.82 & $(0.49,1.36)$ \\
\hline $400 \%$ or greater of FPL & 0.51 & $(0.32,0.83)$ & 0.40 & $(0.26,0.60)$ & 0.52 & $(0.32,0.84)$ \\
\hline \multicolumn{7}{|l|}{ Urban or rural residence } \\
\hline Rural & Reference & & Reference & & Reference & \\
\hline Urban & 1.18 & $(0.79,1.78)$ & 1.17 & $(0.78,1.77)$ & 1.19 & $(0.79,1.80)$ \\
\hline \multicolumn{7}{|l|}{ Health status } \\
\hline Excellent, very good, or good & Reference & & Reference & & Reference & \\
\hline \multirow{2}{*}{\multicolumn{7}{|c|}{ Chronic condition }} \\
\hline & & & & & & \\
\hline No & Reference & & Reference & & Reference & \\
\hline Yes & 1.36 & $(1.02,1.81)$ & 1.40 & $(1.06,1.85)$ & 1.38 & $(1.04,1.83)$ \\
\hline Survey year & & & & & & \\
\hline 2015 & Reference & & Reference & & Reference & \\
\hline 2016. & 1.12 & $(0.85,1.46)$ & 1.12 & $(0.86,1.47)$ & 1.11 & $(0.85,1.47)$ \\
\hline Test for interaction with race & Type of in & ince & Location o & sual source & & \\
\hline & $F$ & $P$ value & & $P$ value & & \\
\hline & 1.55 & 0.0635 & 0.83 & 0.6541 & & \\
\hline
\end{tabular}

Significant associations denoted in italics, at $p<.05$.

AOR adjusted odds ratio, 95\% CI 95\% confidence interval

implementation, it did not factor changing demand for emergency services. In particular, the overall rate of emergency department usage increased post-ACA and there was a shift towards Medicaid paying for this service. ${ }^{34}$ Thus, the increasing use of emergency room care may have worsened overall patient experience with health care in this setting, including experiences of racial or ethnic discrimination.

This study showed that patients with certain types of health insurance are more likely to perceive discrimination. Namely, those with insurance financed through federal programs (i.e., 
Table 3 Logistic Regression Models for Delaying or Forgoing Prescriptions or Medical Care in the Past 12 Months $(N=39,171)$

\begin{tabular}{|c|c|c|c|c|}
\hline \multirow[t]{2}{*}{ Outcome } & \multicolumn{2}{|c|}{$\begin{array}{l}\text { Delayed or did not get needed } \\
\text { prescription in past } 12 \text { months }\end{array}$} & \multicolumn{2}{|c|}{$\begin{array}{l}\text { Delayed or did not get needed } \\
\text { medical care in past } 12 \text { months }\end{array}$} \\
\hline & AOR & $95 \% \mathrm{CI}$ & AOR & $95 \% \mathrm{CI}$ \\
\hline \multicolumn{5}{|c|}{ Experienced racial or ethnic discrimination in health care } \\
\hline No & Reference & & Reference & \\
\hline Yes & 1.97 & $(1.26,3.09)$ & 1.84 & $(1.31,2.59)$ \\
\hline \multicolumn{5}{|l|}{ Type of insurance coverage } \\
\hline Employer-sponsored coverage & Reference & & Reference & \\
\hline Medicaid & 1.14 & $(0.79,1.66)$ & 1.16 & $(0.74,1.82)$ \\
\hline Private coverage, Covered California & 1.01 & $(0.54,1.89)$ & 0.54 & $(0.32,1.92)$ \\
\hline Private coverage, off-exchange & 0.93 & $(0.53,1.61)$ & 0.97 & $(0.59,1.59)$ \\
\hline Medicare and Medicaid & 1.24 & $(0.74,2.06)$ & 1.03 & $(0.63,1.69)$ \\
\hline Medicare alone or with other insurance & 0.81 & $(0.52,1.25)$ & 0.74 & $(0.49,1.12)$ \\
\hline Other public-sponsored coverage & 0.73 & $(0.29,1.80)$ & 1.47 & $(0.75,2.88)$ \\
\hline \multicolumn{5}{|l|}{ Location of usual source of care } \\
\hline Doctor's office, HMO, or Kaiser & Reference & & Reference & \\
\hline No usual source of health care & 1.06 & $(0.71,1.57)$ & 1.17 & $(0.81,1.70)$ \\
\hline Clinic or health center & 1.08 & $(0.81,1.46)$ & 1.28 & $(0.96,1.71)$ \\
\hline Emergency room & 0.82 & $(0.42,1.63)$ & 2.16 & $(1.26,3.73)$ \\
\hline Other or no one place & 2.36 & $(0.93,5.99)$ & 1.53 & $(0.63,3.70)$ \\
\hline Race/ethnicity & - & - & & \\
\hline White & Reference & & Reference & \\
\hline Latino & 0.60 & $(0.44,0.82)$ & 0.62 & $(0.46,0.84)$ \\
\hline Black & 0.76 & $(0.52,1.11)$ & 0.50 & $(0.32,0.78)$ \\
\hline Asian & 0.67 & $(0.45,1.02)$ & 0.58 & $(0.38,0.89)$ \\
\hline Other & 1.15 & $(0.74,1.79)$ & 0.80 & $(0.55,1.17)$ \\
\hline \multicolumn{5}{|l|}{ Citizenship status } \\
\hline US-born citizen & Reference & & Reference & \\
\hline Naturalized citizen & 1.02 & $(0.70,1.47)$ & 0.97 & $(0.68,1.40)$ \\
\hline Non-citizen & 0.58 & $(0.35,0.97)$ & 0.67 & $(0.36,1.25)$ \\
\hline \multicolumn{5}{|l|}{ Limited English proficiency } \\
\hline No & Reference & & Reference & \\
\hline Yes & 0.66 & $(0.42,1.03)$ & 0.62 & $(0.38,1.00)$ \\
\hline \multicolumn{5}{|l|}{ Gender } \\
\hline Male & Reference & & Reference & \\
\hline Female & 1.34 & $(1.07,1.67)$ & 1.32 & $(1.04,1.69)$ \\
\hline \multicolumn{5}{|l|}{ Educational attainment } \\
\hline Less than bachelor's degree & Reference & & Reference & \\
\hline Bachelor's degree or more & 1.16 & $(0.91,1.47)$ & 1.33 & $(1.05,1.75)$ \\
\hline \multicolumn{5}{|l|}{ Age } \\
\hline $18-29$ & Reference & & Reference & \\
\hline $30-39$ & 0.77 & $(0.55,1.09)$ & 0.85 & $(0.59,1.23)$ \\
\hline $40-49$ & 0.95 & $(0.61,1.47)$ & 1.23 & $(0.86,1.76)$ \\
\hline $50-59$ & 0.85 & $(0.60,1.21)$ & 1.00 & $(0.63,1.57)$ \\
\hline $60-69$ & 0.72 & $(0.46,1.12)$ & 0.72 & $(0.48,1.07)$ \\
\hline $70+$ & 0.62 & $(0.34,1.14)$ & 0.47 & $(0.28,0.78)$ \\
\hline \multicolumn{5}{|c|}{ Household income (as \% of federal poverty level (FPL)) } \\
\hline $0-138 \%$ of FPL & Reference & & Reference & \\
\hline $139-249 \%$ of FPL & 0.95 & $(0.65,1.38)$ & 0.85 & $(0.61,1.18)$ \\
\hline $250-399 \%$ of FPL & 1.12 & $(0.75,1.67)$ & 1.10 & $(0.68,1.78)$ \\
\hline $400 \%$ or greater of FPL & 0.99 & $(0.68,1.45)$ & 0.91 & $(0.62,1.35)$ \\
\hline \multicolumn{5}{|l|}{ Urban or rural residence } \\
\hline Rural & Reference & & Reference & \\
\hline Urban & 1.18 & $(0.87,1.59)$ & 1.20 & $(0.89,1.62)$ \\
\hline \multicolumn{5}{|l|}{ Health status } \\
\hline Excellent, very good, or good & Reference & & Reference & \\
\hline Fair or Poor & 1.62 & $(1.25,2.10)$ & 1.89 & $(1.46,2.45)$ \\
\hline \multicolumn{5}{|l|}{ Chronic condition } \\
\hline No & Reference & & Reference & \\
\hline Yes & 1.30 & $(1.03,1.64)$ & 1.14 & $(0.93,1.39)$ \\
\hline \multicolumn{5}{|l|}{ Survey year } \\
\hline 2015 & Reference & & Reference & \\
\hline 2016 & 0.93 & $(0.76,1.14)$ & 0.97 & $(0.78,1.22)$ \\
\hline
\end{tabular}

Significant associations denoted in italics, at $p<.05$.

AOR adjusted odds ratio, $95 \%$ CI $95 \%$ confidence interval

Medicare and Medicaid), relative to employer-sponsored coverage. This suggests that patients in these federal programs have either a higher prior burden of discrimination in healthcare or are exposed to more discrimination because of their federally funded coverage. Controlling for location of usual source of health care did not impact this relationship. The disadvantage experienced by those with Medicaid and Medicare coverage may stem from a variety of factors, including having a harder time getting an appointment, ${ }^{28}$ narrower provider networks, different services covered, longer patient wait- 
times, cost-sharing between patient and insurance, and receiving poorer service from health care providers and their staff based on the insurance they utilize. Thus, both interpersonal and structural factors may be at play. However, with CHIS data, we cannot gage the motives or attributions of those perpetrating the unfair treatment, or determine if a respondent is attributing his or her unfair treatment to race when it is instead due to the features and limitations of their insurance coverage. In any case, our study suggests that providers should work to better understand the factors that lead to discrimination among their patients, particularly among those on publicly funded health insurance. This is important because experiences of racial or ethnic discrimination are associated with lower trust of health care providers, lower trust of the health care system, poorer medication adherence, and worse health. ${ }^{35-37}$

This study also examined the impact of insurance type and location of usual source of health care among different racial/ ethnic groups. There were no significant interactions between either variable and race. This suggests that the impact of perceived discrimination matters, in the same way, irrespective of race. This is important in the case of type of insurance coverage since the ACA brought about new coverage options, with different access to provider networks. Specifically, those with insurance coverage purchased through Covered California have access to a narrower network of hospital providers ${ }^{38}$; however, evidence on the impact these narrower networks have had on quality of health care has been mixed. ${ }^{28,38,39}$ Our findings suggest that new options offered under Covered California did not differentially expose different racial or ethnic groups to racial or ethnic discrimination, relative to private coverage purchased off-exchange. This is important because the Covered California exchanges will undertake efforts to improve quality, including paying hospitals in Covered California's network at lower rates for their services if they fail to meet quality standards, and eventually, eliminating poorer performing and costlier hospitals and providers from their networks. ${ }^{40,41}$

Finally, this study showed that perceived racial or ethnic discrimination was associated with delaying or forgoing needed prescriptions or medical care in the past 12 months. Earlier work had only showed that perceived discrimination was associated with higher odds of delaying a prescription. ${ }^{11}$ The discrepancy in findings may be due to several methodological differences between this and the prior study including a representative study design, racially diverse sample, and much larger sample size. Also, unlike prior work, the present study was able to specifically exclude costs and lack of health insurance as reasons for delaying or forgoing care. As a result, these potential reasons are accounted for. Therefore, we posit that experiences of racial and ethnic discrimination in health care make a person less likely to have a future interaction with health care, and thus less likely to fill a prescription or receive medical care.

Several limitations must be considered when interpreting the findings of the current study. First, because the data are cross-sectional, temporality cannot be determined. Second, all measures are self-reported, which means reporting bias cannot be eliminated. Third, because the data come from only one state, results may not generalize to other states. Fourth, the measure of discrimination is based on perceptions of discrimination, which relies on respondent's subjective interpretation of events. Nonetheless, perceived discrimination is robustly associated with both physical and mental health, suggesting this measure is still important to consider. ${ }^{42}$

Overall, findings suggest the burden of discrimination is unequally distributed in the post-ACA landscape. In particular, those with publicly funded coverage are more likely to perceive discrimination in health care, suggesting that providers and insurers must adapt to deal with the increased burden among their patient pools and make efforts to reduce discrimination of patients they serve. This is particularly important because Medicare and Medicaid are designed to serve populations that are sicker or have lower incomes, respectively. As a result, experiences of discrimination in the health care setting may disproportionately impact those that are already otherwise disadvantaged. Furthermore, because those with prior discrimination are less likely to obtain care or prescriptions, these experiences can create a feedback loop that fuels health disparities.

Corresponding Author: Héctor E. Alcalá, MPH, PhD; Department of Family, Population and Preventive Medicine, Program in Public HealthStony Brook University, Stony Brook, NY, USA (e-mail: hectorapm@ucla.edu).

\section{Compliance with Ethical Standards:}

Conflict of Interest: The authors declare that they do not have a conflict of interest.

\section{REFERENCES}

1. Shavers VL, Fagan P, Jones D, et al. The State of Research on Racial/ Ethnic Discrimination in The Receipt of Health Care. Am J Public Health 2012;102(5):953-966.

2. Harris R, Tobias $\mathbf{M}$, Jeffreys $\mathbf{M}$, Waldegrave $\mathbf{K}$, Karlsen $\mathbf{S}$, Nazroo J. Racism and health: The relationship between experience of racial discrimination and health in New Zealand. Soc Sci Med 2006;63(6): 1428-1441.

3. Stepanikova I, Baker EH, Simoni ZR, et al. The Role of Perceived Discrimination in Obesity Among African Americans. Am J Prev Med 2017;52(1):S77-S85.

4. Ayotte BJ, Hausmann LR, Whittle J, Kressin NR. The relationship between perceived discrimination and coronary artery obstruction. Am Heart J 2012;163(4):677-683.

5. Krieger N, Jahn JL, Waterman PD. Jim Crow and estrogen-receptornegative breast cancer: US-born black and white non-Hispanic women, 1992-2012. Cancer Causes Control 2017;28(1):49-59.

6. Chae DH, Lincoln KD, Adler NE, Syme SL. Do experiences of racial discrimination predict cardiovascular disease among African American men? The moderating role of internalized negative racial group attitudes. Soc Sci Med (1982). 2010;71(6):1182-1188.

7. Myers HF. Ethnicity- and socio-economic status-related stresses in context: an integrative review and conceptual model. J Behav Med 2009;32(1):9-19.

8. Williams DR, Mohammed SA. Discrimination and racial disparities in health: evidence and needed research. J Behav Med 2009;32(1):20-47.

9. Lee C, Ayers SL, Kronenfeld JJ. The Association between Perceived Provider Discrimination, Health Care Utilization, and Health Status in Racial and Ethnic Minorities. Ethn Dis 2009;19(3):330-337. 
10. Hausmann LRM, Jeong K, Bost JE, Ibrahim SA. Perceived Discrimination in Health Care and Use of Preventive Health Services. J Gen Intern Med 2008;23(10):1679-1684.

11. Van Houtven CH, Voils CI, Oddone EZ, et al. Perceived Discrimination and Reported Delay of Pharmacy Prescriptions and Medical Tests. J Gen Intern Med 2005;20(7):578-583.

12. Burgess DJ, Ding Y, Hargreaves M, van Ryn M, Phelan S. The association between perceived discrimination and underutilization of needed medical and mental health care in a multi-ethnic community sample. J Health Care Poor Underserved 2008;19(3):894-911.

13. Mouton CP, Carter-Nolan PL, Makambi KH, et al. Impact of Perceived Racial Discrimination on Health Screening in Black Women. J Health Care Poor Underserved 2010;21(1):287-300.

14. Hausmann LR, Kressin NR, Hanusa BH, Ibrahim SA. Perceived racial discrimination in health care and its association with patients' healthcare experiences: does the measure matter? Ethn Dis 2010;20(1):40.

15. Boulware LE, Cooper LA, Ratner LE, LaVeist TA, Powe NR. Race and Trust in the Health Care System. Public Health Rep 2003;118(4):358 365.

16. Stepanikova I, Oates GR. Perceived Discrimination and Privilege in Health Care: The Role of Socioeconomic Status and Race. Am J Prev Med 2017;52(1):S86-S94.

17. Abramson CM, Hashemi M, Sánchez-Jankowski M. Perceived discrimination in U.S. healthcare: Charting the effects of key social characteristics within and across racial groups. Prev Med Rep 2015;2:615-621.

18. Vina ER, Hausmann LRM, Utset TO, Masi CM, Liang KP, Kwoh CK. Perceptions of racism in healthcare among patients with systemic lupus erythematosus: a cross-sectional study. Lupus Sci Med 2015;2(1):e000110.

19. Hausmann LRM, Jeong K, Bost JE, Kressin NR, Ibrahim SA. Perceived Racial Discrimination in Health Care: A Comparison of Veterans Affairs and Other Patients. Am J Public Health 2009;99(Suppl 3):S718-S724.

20. Sorkin DH, Ngo-Metzger Q, De Alba I. Racial/Ethnic Discrimination in Health Care: Impact on Perceived Quality of Care. J Gen Intern Med 2010;25(5):390-396.

21. Hausmann LRM, Kwoh CK, Hannon MJ, Ibrahim SA. Perceived Racia Discrimination in Health Care and Race Differences in Physician Trust. Race Soc Probl 2013;5(2):113-120.

22. Kandula NR, Hasnain-Wynia R, Thompson JA, Brown ER, Baker DW. Association Between Prior Experiences of Discrimination and Patients Attitudes Towards Health Care Providers Collecting Information About Race and Ethnicity. J Gen Intern Med 2009;24(7):789-794.

23. D'Anna LH, Stevens GD, Malotte CK, Tsai K-Y. Does Health Care Setting Matter in Reports of Discrimination? J Ambul Care Manage 2010;33(4):314-327.

24. Courtemanche C, Marton J, Ukert B, Yelowitz A, Zapata D. Early Impacts of the Affordable Care Act on Health Insurance Coverage in Medicaid Expansion and Non-Expansion States. J Policy Anal Manage 2017;36(1): 178-210.

25. Blumenthal D, Collins SR. Health Care Coverage under the Affordable Care Act — A Progress Report. New Engl J Med 2014;371(3):275-281.
26. Carman KG, Eibner C, Paddock SM. Trends In Health Insurance Enrollment, 2013-15. Health Aff (Millwood) 2015;34(6):1044-1048.

27. Sommers BD, Musco T, Finegold K, Gunja MZ, Burke A, McDowell AM. Health Reform and Changes in Health Insurance Coverage in 2014 N Engl J Med 2014;371(9):867-874.

28. Alcalá HE, Roby DH, Grande DT, McKenna RM, Ortega AN. Insurance Type and Access to Health Care Providers and Appointments Under the Affordable Care Act. Med Care. 2017.

29. Sen AP, Chen LM, Cox DF, Epstein AM. Most Marketplace Plans Included At Least 25 Percent Of Local-Area Physicians, But Enrollment Disparities Remained. Health Aff (Millwood) 2017;36(9):1615-1623.

30. Sommers BD, Kenney GM, Epstein AM. New evidence on the Affordable Care Act: coverage impacts of early medicaid expansions. Health Aff (Millwood) 2014;33(1):78-87.

31. Wehby GL, Lyu W. The Impact of the ACA Medicaid Expansions on Health Insurance Coverage through 2015 and Coverage Disparities by Age, Race/Ethnicity, and Gender. Health Serv Res. n/a-n/a.

32. Chen J, Vargas-Bustamante A, Mortensen $\mathbf{K}$, Ortega AN. Racial and ethnic disparities in health care access and utilization under the Affordable Care Act. Med Care 2016;54(2): 140

33. California Health Interview Survey. Report 1: Sample Design. Los Angeles: UCLA Center for Health Policy Research; 2016.

34. Barakat MT, Mithal A, Huang RJ, et al. Affordable Care Act and healthcare delivery: A comparison of California and Florida hospitals and emergency departments. PLoS One 2017;12(8):e0182346.

35. Cuffee YL, Hargraves JL, Rosal M, et al. Reported racial discrimination, trust in physicians, and medication adherence among inner-city African Americans with hypertension. Am J Public Health 2013;103(11):e55-e62.

36. Armstrong $\mathbf{K}$, Putt $\mathbf{M}$, Halbert $\mathbf{C H}$, et al. Prior Experiences of Racial Discrimination and Racial Differences in Health Care System Distrust. Med Care 2013;51(2):144-150.

37. Gee GC, Ponce N. Associations Between Racial Discrimination, Limited English Proficiency, and Health-Related Quality of Life Among 6 Asian Ethnic Groups in California. Am J Public Health 2010;100(5):888-895.

38. Haeder SF, Weimer DL, Mukamel DB. California hospital networks are narrower in Marketplace than in commercial plans, but access and quality are similar. Health Aff (Millwood) 2015;34(5):741-748.

39. Haeder SF, Weimer DL, Mukamel DB. Secret Shoppers Find Access To Providers And Network Accuracy Lacking For Those In Marketplace And Commercial Plans. Health Aff (Millwood) 2016;35(7):1160-1166.

40. Terhune C. Covered California aims to kick out poor-performing hospitals. 2016. http://www.scpr.org/news/2016/03/18/58667/covered-californiaaims-to-kick-out-poor-performin/. Accessed 22 June 2017.

41. Ibarra AB, Gorn D. Covered California Imposes New Quality, Cost Conditions On Health Plans. 2016. https://ww2.kqed.org/stateofhealth/2016/04/08/covered-california-imposes-new-quality-cost-conditions-on-health-plans/. Accessed 27 Dec 2017.

42. Pascoe EA, Smart RL. Perceived discrimination and health: a metaanalytic review. Psychol Bull 2009;135(4):531. 